\title{
Mesenchymal Stromal Cells in the Bone Marrow Niche Consist of Multi-Populations With Distinct Transcriptional and Epigenetic Properties
}

\section{Sanshiro Kanazawa \\ University of Tokyo \\ Hironori Hojo \\ University of Tokyo \\ Shinsuke Ohba \\ Nagasaki University \\ Junichi Iwata}

The University of Texas Health Science Center at Houston

\section{Makoto Komura}

University of Tokyo

Atsuhiko Hikita

University of Tokyo

Kazuto Hoshi ( $\nabla$ pochi-tky@umin.net )

University of Tokyo

\section{Research Article}

Keywords: MSCs, PDGFR, ATAC, cells

Posted Date: January 13th, 2021

DOl: https://doi.org/10.21203/rs.3.rs-138736/v1

License: (9) This work is licensed under a Creative Commons Attribution 4.0 International License.

Read Full License

Version of Record: A version of this preprint was published at Scientific Reports on August 4th, 2021. See the published version at https://doi.org/10.1038/s41598-021-94186-5. 


\section{Abstract}

Although multiple studies have investigated the mesenchymal stem and progenitor cells (MSCs) that give rise to mature bone marrow, high heterogeneity in their morphologies and properties causes difficulties in molecular separation of their distinct populations. In this study, by taking advantage of the resolution of the single cell transcriptome, we analyzed Sca-1 and PDGFR-a fraction in the mouse bone marrow tissue. The single cell transcriptome enabled us to further classify the population into seven populations according to their gene expression profiles. We then separately obtained the seven populations based on candidate marker genes, and specified their gene expression properties and epigenetic landscape by ATAC-seq. Our findings will enable to elucidate the stem cell niche signal in the bone marrow microenvironment, reconstitute bone marrow in vitro, and shed light on the potentially important role of identified subpopulation in various clinical applications to the treatment of bone- and bone marrowrelated diseases.

\section{Introduction}

The bone marrow microenvironment, which is a multifunctional network between cells and the extracellular matrix, plays crucial roles in maintenance of proliferation, differentiation, and survival of stromal cells and hematopoietic cells ${ }^{1}$. One important cell lineage derived from bone marrow is mesenchymal stem/progenitor cells (MSCs). These cells have the ability to differentiate into various lineage such as osteocytes, chondrocytes, adipocytes, etc., corresponding to external signals from the microenvironment and endogenous ones inducing commitment of cell lineages.

Among various kinds of cells in the bone marrow, hematopoietic stem cells (HSCs) have been intensively studied. The research field has been progressed because markers for isolating HSCs have been identified. CD34-/KLS and CD34-KLS/CD150 + have been defined as markers for HSCs by international society for stem cell research ${ }^{2,3}$. About 1000 to 3000 HSCs can be harvested per mouse at almost $100 \%$ purity, while differentiation and cell death taking place during collection decrease the purity of HSCs about 20 to $50 \%{ }^{4}$. Many studies are also conducted on $\mathrm{MSCs}^{5-8}$. Several combinations of markers have been discovered for isolating MSCs, and functions of the isolated cells were evaluated ${ }^{9-12}$. However, cells expressing these markers are still composed of heterogeneous cell populations, which hinders precise characterization of MSCs ${ }^{13-21}$. For example, Sca-1 (Ly6 A / E) and PDGFR-a (Pa-S) are currently considered to be useful for the MSCs separation ${ }^{22}$. However, the cells separated using these markers contain heterogenous progenitor populations, within which bona-fide MSCs exist because part of these cells express Nestin, NG2 or leptin receptor in vivo ${ }^{23}$, which is considered as a MSC marker ${ }^{24}$. In addition, pericytes expressing Tbx18 are also positive for MSC marker such as CD146 or Sca-1, indicating the heterogeneity of Pa-S population ${ }^{15}$. To understand the nature of MSCs more profoundly, it is quite important to dissect the heterogenous populations of $\mathrm{Pa}-\mathrm{S}$, and analyze each population separately. 
In this study, we identified subpopulations in mouse bone marrow MSCs positive for Pa-S by single-cell level with single-cell RNA-seq (scRNA-seq) analysis. Assay for Transposase Accessible Chromatin with sequencing (ATAC-seq) analysis of each of the subpopulations further revealed epigenetic landscape, their genetic similarities and functional properties. Our findings will contribute to the elucidation of the essential characteristics of MSCs, which lead to the safe and effective application of the cells to clinical settings.

\section{Results}

\section{Identification of heterogenic cell population in isolated MSCs using scRNA-seq}

Currently, selection markers useful for isolating bone marrow-derived stem/ progenitor cells (MSCs) with advances in FACS technology dramatically improve purification of the cell population compared to conventional methods ${ }^{25}$. As a consequence, the analysis of MSC's properties had significantly progressed, and the immunosuppression ${ }^{18}$ and anti-inflammatory capacity of MSCs with multipotency have been shown ${ }^{26,27}$. However, since more than half of the cells isolated as MSCs are heterogenic cell population, it remains unknown whether they are the main function of MSCs. Therefore, it is important to clarify what kind of cell population the isolated MSCs have. To answer these questions, we first performed scRNA-seq using a platform of Chromium Controller (10x Genomics, Inc) in 30,000 of MSCs ${ }^{28}$, which were negative for CD31, 45, and Ter119 and positive for Sca-1 and Pdgfr-a (Fig. 1, a). As a result, we found that the seven different cell subpopulations exist in isolated MSCs according to genetic similarities (Fig. 1, b); each of these subpopulations showed distinct gene expression (Fig. 1, c and Supplemental information Fig 1). Annotation analysis with the profile suggest that cluster 1 may have osteogenic and endothelial differentiation properties, clusters 2 and 5 may have osteogenic/ chondrogenic ones, cluster 6 may have adipogenic directionality, and clusters 3 and 4 showed characteristic of the hematopoietic lineage. Furthermore, we found functional features of the clusters based on expressed genes: cluster 1, cell aging; cluster 3, cell cycle; cluster 4, biological defense; and cluster 7, transcriptional/ translational regulation. These results indicate that cells isolated as MSCs in the previous research are a heterogeneous cell population where every member shows distinct gene expression properties.

\section{Identification of molecular markers enabling to isolate each of populations}

To identify molecular markers enabling us to sort each of the identified populations, we listed candidate markers that are available for FACS sorting among the genes of which expressions showed a 1.5-fold or more increase or decrease in scRNA-seq (Fig 1c, Supplemental information Fig. 2). As a result, we found that most of the populations had common candidate marker genes CD24, 39, 54, Sca-1 and Ly-6C. When these were used together or different combination to isolate them, subpopulation could be separated at a rate of 0.02 to $1.12 \%$ of the total population (Fig. 2). Thus, the selection markers that we identified here were useful for isolation of individual subpopulations. 


\section{Genetic character in each cluster by scRNA-seq and ATAC-seq}

To evaluate the genetic characteristics of the subpopulations identified by scRNA-seq, chromatin landscape and gene expression profiles were examined in each of the sorted population (clusters 1 to 7 ) by ATAC-seq and RNA-seq analysis, respectively. Based on the peak intensity of ATAC-seq, we conducted clustering analysis and made a PCA plot. The seven clusters were largely divided into two groups according to similarities of open chromatin signatures: group 1 comprised of clusters 1, 3, 4, and 7; group 2 comprised of clusters 2,5 , and 6 . In the group 1 , cluster 4 was most similar to cluster 1 , and then similar to clusters 3 and 7 (Fig. 3 a, b). When gene expression data obtained by RNA-seq were also subjected to clustering analysis and PCA plot analysis, we obtained similar results to the open chromatin signature; cluster 4 was most similar to cluster 1, and then similar to clusters 3 and 7 , suggesting that the subpopulations identified by scRNA-seq were successfully sorted (Fig. 3 c, d). These data indicate that each cluster is an independent population, along with genetic similarity between the clusters to some extent.

\section{Clusters possess the closest properties to stem cells}

To characterize each of the subpopulations functionally, we analyzed their CFU-F activities and differentiation potentials in vitro. Most of the subpopulations had the CFU-F activity as in the Pa-S group, while clusters 1 and 5 had significantly higher activity (Fig. 4 a). Cluster 1 and 5 also showed vigorous proliferation in the cell proliferation assay (Fig. 4 b). Cluster 3 and 4 proliferated at similar rate as PaS, while cluster 2, 6 and 7 showed much poorer proliferations (Fig. 4 b). Furthermore, when these subpopulations were induced trilineage differentiation, each cluster showed peculiar pattern of the differentiation ability (Fig. 4 c). However, there was no significant difference in the gene expression, because of high batch to batch variation. Cluster 1 and 5, which showed significantly higher CFU activity (Figure 4 a), tended to differentiate into osteogenic and adipogenic, but not chondrogenic linages. The cluster 4,1 of the 2 clusters which showed comparable proliferation ability to PaS, showed tendency of higher expression of 5 genes out of 6 examined, and similar expression of Sox 9 compared to PaS. These results suggested that cluster 4 would possess the closest properties to stem cells, and clusters 1 and 5 might also have similar properties.

\section{Functional significance in open chromatin regions by GREAT analysis}

Given the stem cell-like characteristics of cluster 4, we next attempted to identify cluster 4-specific signatures. However, in comparison of ATAC-seq and RNA-seq data between all clusters, we found no cluster 4-specific open chromatin regions or expressed genes, although there is a similarity between open chromatin and the signature of gene expression (Fig. 3 b, c). We then focused on clusters 1, 4, and 5, of which properties were similar to stem cells, and compared their ATAC-seq peaks. Among the three clusters, cluster 1 and cluster 4 showed similar gene expression profile and open chromatin signature, while cluster 4 seemed to be closer to stem cells. Therefore, at first, we focused on open chromatin regions that were present in clusters 4 , but not in cluster 1 , as these regions may be associated with stem cell property of cluster 4; we performed GREAT analysis on these regions to investigate their functional 
significance (supplemental Figure 2). The regions were associated with genes expressed in oocytes or pre-implantation embryos (secondary polar body, zona pellucida and compacted morula), indicating that the cluster 4-associated genes may represent immature stem cell property of the cluster. Furthermore, we compared the open chromatin regions of cluster 4 and 5 , because tri-linage differentiation assay also indicated more stem-cell like properties of cluster 4 (Figure 4). Surprisingly, these 2 clusters shared the open chromatin regions indicating immature characteristics describe above (supplemental Figure 3 ). From 576 open chromatin regions that were commonly present in clusters 4 and 5, but not in cluster 1 in the Venn diagram, we searched for open chromatin regions specific for clusters 4 that represented stem cell properties (Figure 5). However, it was difficult to precisely evaluate factors that represent stem cell properties such as immature and differentiation tropism, and that were expressed in a cluster-specific manner.

\section{Discussion}

Recently, genome structure has been known to play an important role in stem cell property. Previous studies for ES cells and iPS cells reported that genomic regions involved in differentiation-directed regulation undergoes epigenetic regulation by methylation or acetylation at a certain stage ${ }^{29}$. Regarding tissue stem cells, epigenomic analyses of HSC revealed that undifferentiated state was maintained by the polycomb complex protein ${ }^{30}$.

However, analysis of genome structure has not progressed for tissue stem cells such as MSCs because of the heterogeneity of cells collected using existing markers for MSCs. We hypothesized that if cells with different functions and characteristics existing in MSC could be individually separated, it is possible to elucidate the innate tissue stem cell, which is clearly different from previous studies. To verify the hypothesis, we took advantage of scRNA-sEq. NGS technology has been dramatically improved in recent years, and it is now possible to perform detailed genetic analysis at the single-cell level for heterogeneous cell population including stem, progenitor and stroma cells, which were previously thought to be MSCs.

Our scRNA-seq analysis revealed seven genetically distinct subpopulations in the mesenchymal stromal cell population. We then found genes that are mutually expressed in these cell populations by gene expression profiles and successfully separated cell populations individually by combination of previously established MSC markers CD24, 39, 54, and Sca-1. Therefore, in this study, we also confirm that these genes are useful markers to isolate subpopulations ${ }^{5,14,31-39}$.

Next, we hypothesized that the change of genome structure plays a key role for MSC properties, and we expected that inventive results could be obtained by ATAC-seq analysis in bone marrow-derived MSCs. This study provided the first molecular characterization and functional follow-up of these seven cell populations. Gene expression profiles in subdivided individual clusters and epigenome data obtained by our ATAC-seq analysis suggest potential mechanisms underlying the specification of each of these populations. Selective detection of the open chromatin structure on a genome-wide basis enables to 
elucidate the mechanism of gene regulation. As a result, the specificity of each cluster indicated an independent cell population from heterogenous cell population ${ }^{40}$.

Our in vitro studies suggest that clusters have functionally different properties, and bioinfomatic analysis on RNA-seq and ATAC-seq also confirmed the properties of each cluster. Therefore, we demonstrated profile of the genes by RNA-seq and ATAC-seq, and found that each cluster in MSCs has a distinct transcriptome and epigenome. Thus, our study directly addressed the question of how each cluster has independent features, identifying many known regulators that are potentially involved in the establishment of cell-type specific chromatin structures and gene expression programs.

ATAC-seq contained more information to identify cluster type-specific features. Indeed, our data showed that the expression of cell type-specific genes was well correlated with the chromatin accessibility of the corresponding promoters; the gene expression pattern in RNA-seq and open chromatin signature in ATACseq are in a good agreement. ATAC-seq can identify not only promoter regions, but also the open chromatin regions of intergenic regions, which presumably contain cell-type specific enhancer regions ${ }^{41}$. However, we failed to find cluster-specific open chromatin regions, as in stem cells characterized by differentiation directionality regulation and maintenance of immature. Given that higher order structure of the genome including the open chromatin status is set up prior to gene expression and not strictly specific for each cell types ${ }^{42}$, open chromatin is not necessarily associated with subpopulation-specific gene expression in MSCs. In addition, we could not confirm specific gene expression patterns that precisely characterize each of the clusters, suggesting that we focused on the stem cell properties.

In this study, we attempted to identify tissue stem cells in the bone marrow; our analysis did not detect previously reported ES- and iPS-like population in MSCs. However, our GREAT analysis indicates that clusters plays an important role in organogenesis in early development. Thus, these results suggest that isolated clusters are available for treatment of tissues defects in bone and cartilage diseases. Further examination will require on whether or not the clusters have pulripotency.

\section{Methods}

\section{Mice}

All experimental procedures and protocols for the present experiments were approved by the ethics committee or institutional committee for animal research of the University of Tokyo and were performed in accordance with the Guide for the Care and Use of Laboratory Animals and the ARRIVE guidelines. C57BL/6JJcl mice (6-12 weeks old) were purchased from CLEA Japan, Inc. (Tokyo, Japan). C57BL/6$\mathrm{Tg}$ (CAG-EGFP)10sb/J mice, transgenic mice that ubiquitously express EGFP under the control of the CAG promoter, 6-12 weeks of age were purchased from CHARLES RIVER LABORATORIES JAPAN, INC. (Yokohama, Japan). The mice were kept under specific pathogen-free conditions in our animal facility at the University of Tokyo.

\section{RNA isolation and quantitative PCR}


RNA isolation of differentiated cells. Live cells were collected in Tri-Reagent buffer (Sigma-Aldrich \#T9424) and cell lysates were homogenized with $21 \mathrm{G}$ needle on ice. Reverse transcription was performed using the PrimeScript RT reagent kit (TaKaRa \#PR0037A), following the manufacturer's recommendations for a standard-yield reaction (15 min of amplification time). mRNA expression was normalized to Hprt1 (for the experiments represented in Fig. 4. Expression levels of mRNA were assessed by real-time PCR using the SYBR Green Master Mix (Thermo Fisher Scientific \#A25743). mRNA expression was normalized to Hprt1 for mouse experiments presented in Fig. 4. Gene expression assays show mean values over $n=3$ biological replicates; the experiment was performed three times.

\section{scRNA-seq}

For the initial scRNA-seq experiment was performed using the Chromium Single Cell Gene Expression Solution (10x Genomics), following the manufacturer's protocol. The MSC was isolated from ten 6-weekold mice (all males). Cells were stained with the anti-mouse antibodies CD31 PE-Cy7, CD45 PE-Cy7 and TER119 PE-Cy7 (Biolegend \#303110, \#304017 and \#116215, respectively), Sca-1 PE (eBioscience \#125981-82) and PDGFR-a APC (eBioscience \#17-1401-81), and 30,000 Lin- Sca-1+ Pdgfr-a+ were isolated using a BD Bioscience FACS Aria III Fusion. Cells were washed and resuspended in $250 \mu$ FACS buffer (PBS, 2\% FBS, 1 mM EDTA), targeting the required 1,000 cells/ $\mu$ l concentration, accounting for a 10-20\% loss. We pipetted $9.7 \mu \mathrm{l}$ cell suspension (concentration of 913 cells/ $\mu \mathrm{l}, \quad 8,800$ cells), targeting the recovery of $\sim 5,000$ cells. Single-cell RNA-seq libraries were obtained following the 10x Genomics recommended protocol, using the reagents included in the Chromium Single Cell 3' v2 Reagent Kit. Libraries were sequenced on the NextSeq 500 v2 (Illumina) instrument using 150 cycles (18 bp barcode + UMI, and 132-bp transcript 3' end), obtaining $\sim 5 \times 108$ raw reads ${ }^{43,44}$.

\section{scRNA-seq data analysis}

The 10x Genomics scRNA-seq data was processed using cellranger-2.1.0, default parameters and the mouse NCBI38/mm10 genome. Molecular counts were obtained for 2,300 cells (filtered matrix), with 160,000 mean reads/cell, an average of $60.5 \%$ reads mapping to the transcriptome and 3,000 median genes detected per cell. We also filtered outlier cells using the median absolute deviation from the median total library size (logarithmic scale) as well as total gene numbers (logarithmic scale), as implemented in scran36, using a cutoff of 3 (isOutlier, nmads $=3$ ). Log (normalized expression) values were obtained using size factors per cell, estimated with scran. The majority of the cells expressed high levels of the Cd24, 39, 54 as well as the MSC marker genes Sca-1 (Supplemetal Data Fig. 1). An initial analysis using all cells revealed that one of the major sources of variation was related to the expression of the gene Xist, as exemplified by the strong clustering of cells expressing Xist in the 2D t-SNE projection (Fig. 1). Genomic alignment rates and number of detected genes/cell suggested that our data were of high quality ${ }^{45}$.

\section{FACS-based cell isolation of mouse cells}


The isolated single-cell suspension was diluted to 0.75 or $1 \times 107$ cells $/ \mathrm{ml}$ with FACS buffer (PBS with $2 \%$ FBS, 1 mM EDTA, 1\% penicillin) and the following antibodies were added: anti-mouse CD31 PE-Cy7, antimouse CD45 PE-Cy7, anti-mouse TER119 PE-Cy7 (BioLegend \#303110, \#304017 and \#116215, respectively) for selecting the Lin- population; anti-mouse Sca1-PE (eBioscience \#12-5981-82), antimouse CD140a APC (eBioscience \#17-1401-81) and anti-mouse CD24 PE, APC (eBiosciences \#12-024742, \#17-0247-42) to enrich the Lin- population with ASPCs; anti-mouse CD39 PE, APC (eBioscience \#123390-80, \#17-0399-42), anti-mouse CD54 PE, APC(eBioscience \#12-0542-82, \#17-0542-82) with APC for separating populations negative and positive for the given marker.

The cells were incubated with the cocktail of antibodies on ice for 20 min protected from light, after which they were washed and stained with DAPI (Sigma \#D9542) or propidium iodide (Molecular Probes \#P3566) for assessing viability, and subjected to FACS using a Becton Dickinson FACSAria III Fusion sorter. Compensation measurements were performed for single stains using compensation beads (eBiosciences \#01-2222-42).

\section{Differentiation cultures}

To induce adipocyte differentiation, subconfluent cells were cultured with 3 cycles of Adipogenic Induction Medium/ Adipogenic Maintenance Medium, with supplements from the Adipogenic Induction/Adipogenic Maintenance SingleQuot kit (Lonza Ltd). Each cycle consisted of feeding the subconfluent cells with the induction medium for 3 days, followed by 3 days of culture in the maintenance medium. After 14 days, the cells were harvested with TRI Reagent (Sigma Aldrich). For chondrogenic differentiation, the $2 \times 10^{4}-2.5 \times 10^{5}$ cells were seeded into a $15-\mathrm{mL}$ conical tube. The tube was spun at $400 \mathrm{~g}$ for 5 minutes at room temperature, and the supernatant was aspirated. The cells were resuspended in $1 \mathrm{~mL}$ Differentiation Basal Medium Chondrogenic, with supplements from the Chondrogenic SingleQuot kit (Lonza Ltd), spun at 400xg for $5 \mathrm{~min}$, and the medium was aspirated. The cells were resuspended in $1 \mathrm{~mL}$ of Differentiation Basal Medium Chondrogenic, supplemented with Chondrogenic SingleQuots kit, TGF- $\beta 3$ (10 ng/mL; Lonza Ltd) and BMP-6 (500 ng/mL; R\&D Systems, Inc), and spun at $150 \mathrm{~g}$ for 5 minutes at room temperature. The pellet was maintained with Differentiation Basal Medium changed every 3-4 days for 2 weeks. After 3 weeks, cell clumps were harvested with TRI Reagent. To induce osteocyte differentiation, subconfluent cells were cultured with Differentiation Basal Medium Osteogenic, supplemented with Osteogenic SingleQuots (Lonza Ltd) for 14 days. The cells were then harvested with TRI Reagent ${ }^{15}$.

\section{Real-Time RT-PCR Analysis for Selected mRNAs}

About $200 \mathrm{ng}$ of total RNA was used to synthesize double-stranded cDNA by reverse transcription (Super Script III; Invitrogen). cDNA was analyzed by real-time PCR using Universal SYBR green PCR Master Mix (Invitrogen). For the assays, reaction mixtures were incubated at $50^{\circ} \mathrm{C}$ for 2 minutes, $95^{\circ} \mathrm{C}$ for 10 minutes, and then 40 cycles of $95^{\circ} \mathrm{C}$ for 15 seconds followed by $60^{\circ} \mathrm{C}$ for 1 minute. For relative quantitation of 
gene expression, mouse-specific Gapdh and Hprt1 (Invitrogen) were used as internal controls. All other PCR primer sequences are listed in the supplementary table.

\section{RNA-seq analysis}

RNA-seq analysis was performed as described ${ }^{46}$. Briefly, 35,000 cells were collected and $30 \mathrm{ng}$ of total RNA was subjected to analysis. The RNA libraries were constructed by mRNA sequencing via polyA selection kit. Sequencing was performed with Hiseq PE 2x150 (Illumina). The sequence reads were aligned and mapped using Partek Flow software v2.2 (Partek). The raw reads were first subjected to prealignment Quality Assurance and Quality Control (QA/QC). Any base below a Phred value of 20 was trimmed from either side of the read and reads shorter than $25 \mathrm{nt}$ length were removed. The processed reads were aligned by Tophat2-2.0.8 ${ }^{47}$ to $\mathrm{mm} 9$ reference genome. The mapping quality and coverage were checked by post-alignment QA/QC. Aligned reads were quantified and normalized as reads per kilo base length of transcript per million reads (RPKM). Heat map of hierarchical clustering and PCA plot were generated by Partek Genomics Suite. In the analysis, we used selected genes based on the following criteria: fold change $>2$ and rpkm $>2$ for the hierarchical clustering; rpkm $>2$ for the PCA plot.

\section{ATAC-seq analysis}

The ATAC-seq was performed as described ${ }^{48}$. Briefly, 50,000 cells were collected and lysed with lysis buffer containing $10 \mathrm{mM}$ Tris- $\mathrm{HCl}, 10 \mathrm{mM} \mathrm{NaCl}, 3 \mathrm{mM} \mathrm{MgCl}$, and $0.1 \%$ IGEPAL CA-630. Tn5 transposase reaction using the Tagment DNA Enzyme 1 (TDE1) (Illumina, catalog \# 15027865) was carried out at $37^{\circ} \mathrm{C}$ for $30 \mathrm{~min}$. The reacted DNA was purified using QIAGEN MinElute PCR purification kit and amplified for 8-15 cycles to produce libraries for sequencing. The ATAC-seq libraries were sequenced on lllumina Hiseq $X$ sequencer. The sequence reads were aligned to the mouse genome reference sequence $\mathrm{mm} 9$ by bowtie aligner ${ }^{49}$. Peak calling was performed by two-sample analysis on CisGenome software ${ }^{50}$ with a Pvalue cutoff of $10^{-5}$ comparing with the input control. Peaks were incorporated into further analysis displaying an FDR $<0.01$. Correlation heat map and PCA plot were generated by DiffBind software in $R$ with default setting. Peak intersection was performed by BEDTools-Version-2.16.2. For gene ontology analysis, GREAT GO analysis was performed utilizing the online Genomic Regions Enrichment of Annotations Tool (GREAT), version 3.0.1 ${ }^{51}$ with default setting.

\section{Statistical Analysis}

The data are presented as the mean \pm standard error of the mean (SEM) and each independent experiment shown was reproduced three to five times. The comparison between two conditions was done by unpaired $t$ test. A one-way repeated-measures ANOVA was applied to identify significant differences among conditions or groups. When a significant difference was observed, the data were subjected to post hoc analysis. A $p<0.05$ was considered significant.

\section{Declarations}


Acknowledgements:

We would like to thank Dan Riu (Department of Tissue Engineering, The University of Tokyo Hospital) for contribution to several experiment. This study was supported in part by Grant-in-Aid for Scientific Research (B) and Grant-in-Aid for Research Activity Start-up of JSPS KAKENHI Grant Number JP18H02998, JP19K21388. Atsuhiko Hikita is Affiliation with an endowed chair from FUJISOFT INCORPORATED.

\section{Author Contribution}

S.K. and K.H. designed the study. S.K., H.H., and S.O. performed the experiment, analyzed the data and interpreted the data. A.H., and M.K. helped with protocol design. K.H., and J.I. supervised the experiment. S.K wrote the manuscript, which was edited by A.H., S.O., and H.H.

\section{References}

1. Lee, K., Majumdar, M. K., Buyaner, D., Hendricks, J. K. \& Pittenger, M. F. J. D. Mosca. Human mesenchymal stem cells maintain transgene expression during expansion and differentiation. Mol. Ther.3, 857-866 https://doi.org/10.1006/mthe.2001.0327 (2001).

2. Osawa, M., Hanada, K., Hamada, H. \& Nakauchi, H. Longterm lymphohematopoietic reconstitution by a single CD 34-low /negative hematopoietic stem cell. Science $273,242-245$. 10.1126/science.273.5272.242. (1996)

3. Morita, Y., Ema, H. \& Nakauchi, H. Heterogeneity and hierarchy within the most primitive hematopoietic stem cell compartment. J. Exp. Med.207, 1173-1182 https://doi.org/10.1084/jem.20091318Epub 2010 Apr 26 (2010).

4. Nakauchi, H., Sudo, K. \& Ema, H. Quantitative assessment of the stem cell self-renewal capacity. Ann. N. Y. Acad. Sci.938, 18-24 discussion 24 - 5 https://doi.org/10.1111/j.1749-6632.2001.tb03570.x (2001).

5. Suto, E. G. et al. Prospectively isolated mesenchymal stem/stromal cells are enriched in the CD73+ population and exhibit efficacy after transplantation. Sci. Rep.7, 4838 https://doi.org/10.1038/s41598-017-05099-1 (2017).

6. Mabuchi, Y. et al. LNGFR(+)THY-1(+)VCAM-1 (hi+) cells reveal functionally distinct subpopulations in mesenchymal stem cells. Stem. Cell. Reports. 1, 152 - 65. 10.1016/j.stemcr.2013.06.001. eCollection 2013. (2013)

7. Cano, E. \& Gebala, V. H. Gerhardt. Pericytes or Mesenchymal Stem Cells: Is That the Question? Cell. Stem. Cell.20, 296-297 https://doi.org/10.1016/j.stem.2017.02.005 (2017).

8. Caplan, A. L. Mesenchymal Stem Cells: Time to Change the Name! Stem. Cells. Transl. Med.6, 14451451 https://doi.org/10.1002/sctm.17-0051Epub 2017 Apr 28 (2005).

9. Cosenza, S., Ruiz, M., Toupet, K., Jorgensen, C. \& Noël, D. Mesenchymal stem cells derived exosomes and microparticles protect cartilage and bone from degradation in osteoarthritis. Sci Rep.7, 16214 
https://doi.org/10.1038/s41598-017-15376-8 (2017).

10. Giri, J., Das, R., Nylen, E., Chinnadurai, R. \& Galipeau, J. CCL2 and CXCL12 derived from mesenchymal stromal cells cooperatively polarize IL-10 + tissue macrophages to mitigate gut injury. Cell. Rep.30, 1923-1934 e4 https://doi.org/10.1016/j.celrep.2020.01.047 (2020).

11. Giuliani, M. et al. J. J. Lataillade. TLR ligands stimulation protects MSC from NK killing. Stem. Cells.32, 290-300 https://doi.org/10.1002/stem.1563 (2014).

12. English, K., Barry, F. P. \& Field-Corbett, C. P. B. P. Mahon. IFN-gamma and TNF-alpha differentially regulate immunomodulation by murine mesenchymal stem cells. Immunol Lett.110, 91-100 https://doi.org/10.1016/j.imlet.2007.04.001Epub 2007 Apr 26 (2007).

13. Pittenger, M. F., Discher, D. E., Péault, B. M., Phinney, D. G. \& Hare, J. M. A. L. Caplan. Mesenchymal stem cell perspective: cell biology to clinical progress. NPJ. Regen. Med. 4. 10.1038/s41536-0190083-6. eCollection 2019. (2019)

14. Sacchetti, B. et al. Self-renewing osteoprogenitors in bone marrow sinusoids can organize a hematopoietic microenvironment. Cell.131, 324-336 https://doi.org/10.1016/j.cell.2007.08.025 (2007).

15. Morikawa, S. et al. Prospective identification, isolation, and systemic transplantation of multipotent mesenchymal stem cells in murine bone marrow. J. Exp. Med.206, 2483-2496 https://doi.org/10.1084/jem.20091046 (2009).

16. Crisan, M. et al. B. Péault. A perivascular origin for mesenchymal stem cells in multiple human organs. Cell. Stem. Cell.3, 301-313 https://doi.org/10.1016/j.stem.2008.07.003 (2008).

17. Méndez-Ferrer, S. et al. P. S. Frenette. Mesenchymal and haematopoietic stem cells form a unique bone marrow niche. Nature.466, 829-834 https://doi.org/10.1038/nature09262 (2010).

18. Salem, H. K. \& Thiemermann, C. Mesenchymal stromal cells: current understanding and clinical status. Stem. Cells.28, 585-596 (2010). 10.1002/stem.269. 10.1016/j.cytogfr.2009.10.002

19. Meirelles Lda, S., Fontes, A. M. \& Covas, D. T. A. L. Caplan. Mechanisms involved in the therapeutic properties of mesenchymal stem cells. Cytokine. Growth. Factor. Rev.20, 419-427 https://doi.org/10.1016/j.cytogfr.2009.10.002 (2009).

20. Nombela-Arrieta, C., Ritz, J. \& Silberstein, L. E. The elusive nature and function of mesenchymal stem cells. Nat. Rev. Mol. Cell. Biol.12, 126-131 https://doi.org/10.1038/nrm3049 (2011).

21. Moretta, L., Uccelli, A. \& Pistoia, V. Mesenchymal stromal cells and immunity: Introductory overview. Immunol. Lett.168, 127-128 https://doi.org/10.1016/j.imlet.2015.08.010 (2015).

22. Koide, Y. et al. Y. Matsuzaki. Two distinct stem cell lineages in murine bone marrow. Stem. Cells.25, 1213-1221 (2007). 10.1634/stemcells.2006 - 0325

23. Matsuzaki, Y., Mabuchi, Y. \& Okano, H. Leptin receptor makes its mark on MSCs. Cell. Stem. Cell.15, 112-114 https://doi.org/10.1016/j.stem.2014.07.001 (2014).

24. Zhou, B. O., Yue, R., Murphy, M. M., Peyer, J. G. \& Morrison, S. J. marrow. Cell. Stem. Cell.15, 154-168 https://doi.org/10.1016/j.stem.2014.06.008 (2014). Leptin-receptor-expressing mesenchymal 
stromal cells represent the main source of bone formed by adult bone

25. Akiyama, K. et al. S. Shi. Mesenchymal-stem-cell-induced immunoregulation involves FASligand-/FAS-mediated T cell apoptosis. Cell. Stem. Cell.10, 44-55 https://doi.org/10.1016/j.stem.2012.03.007 (2012).

26. Yang, R. et al. A subset of IL-17(+) mesenchymal stem cells possesses anti-Candida albicans effect. Cell. Res.23, 107-121 https://doi.org/10.1038/cr.2012.179 (2013).

27. Kudo-Saito, C. et al. M. Toyoura. Blocking the FSTL1-DIP2A axis improves anti-tumor immunity. Cell Rep.24, 1790-1801 https://doi.org/10.1016/j.celrep.2018.07.043 (2018).

28. Houlihan, D. D. et al. Isolation of mouse mesenchymal stem cells on the basis of expression of Sca-1 and PDGFR-a. Nat. Protoc.7, 2103-2111 https://doi.org/10.1038/nprot.2012.125 (2012).

29. Kim, K. et al. G. Q. Daley. Epigenetic memory in induced pluripotent stem cells. Nature.467, 285-290 https://doi.org/10.1038/nature09342 (2012).

30. Oguro, H. et al. Poised lineage specification in multipotential hematopoietic stem and progenitor cells by the polycomb protein Bmi1. Cell. Stem. Cell.6, 279-286 https://doi.org/10.1016/j.stem.2010.01.005 (2010).

31. Lian, Q. et al. S. K. Lim. Derivation of clinically compliant MSCs from CD105+, CD24- differentiated human ESCs. Stem. Cells.25, 425-436 (2007). 10.1634/stemcells.2006 - 0420

32. Schäck, L. M. et al. Expression of CD24 in human bone marrow-derived mesenchymal stromal cells is regulated by TGFß3 and induces a myofibroblast-like genotype. Stem. Cells. Int.1319578, https://doi.org/10.1155/2016/1319578 (2016).

33. Carreras-Planella, L., Monguió-Tortajada, M., Borràs, F. E. \& Franquesa, M. Immunomodulatory effect of MSC on B Cells is independent of secreted extracellular vesicles. Front. Immunol.10, 1288 https://doi.org/10.3389/fimmu.2019.01288 (2019).

34. Saldanha-Araujo, F. et al. R. A. Panepucci. Mesenchymal stromal cells up-regulate CD39 and increase adenosine production to suppress activated T-lymphocytes. Stem. Cell. Res.7, 66-74 https://doi.org/10.1016/j.scr.2011.04.001 (2011).

35. Saldanha-Araujo, F. \& Panepucci, R. A. CD39 expression in mesenchymal stromal cells. J. Immunother.34, 568 https://doi.org/10.1126/science.aav2501 (2011).

36. Merrick, D. et al. Identification of a mesenchymal progenitor cell hierarchy in adipose tissue. Science.364, 6438 https://doi.org/10.1126/science.aav2501 (2019).

37. Espagnolle, N., Balguerie, A., Arnaud, E., Sensebé, L. \& Varin, A. CD54-mediated interaction with proinflammatory macrophages increases the immunosuppressive function of human mesenchymal stromal cells. Stem. Cell. Reports.8, 961-976 https://doi.org/10.1016/j.stemcr.2017.02.008 (2017).

38. Peister, A., Mellad, J. A., Larson, B. L., Hall, B. M. \& Gibson, L. F. D. J. Prockop. Adult stem cells from bone marrow (MSCs) isolated from different strains of inbred mice vary in surface epitopes, rates of proliferation, and differentiation potential. Blood.103, 1662-1668 https://doi.org/10.1182/blood2003-09-3070 (2004). 
39. Niibe, K. et al. Purified mesenchymal stem cells are an efficient source for iPS cell induction. PLoS. One.6, e17610 https://doi.org/10.1371/journal.pone.0017610 (2011).

40. Dirks, R. A., Stunnenberg, H. G. \& Marks, H. Genome-wide epigenomic profiling for biomarker discovery. Clin. Epigenetics. 8, 122. eCollection(2016). 10.1186/s13148-016-0284-4.

41. Ho, Y. T. et al. Chromatin accessibility identifies diversity in mesenchymal stem cells from different tissue origins. Sci. Rep.8, 17765 https://doi.org/10.1038/s41598-018-36057-0 (2018).

42. Klemm, S. L. \& Shipony, Z. W. J. Greenleaf. Chromatin accessibility and the regulatory epigenome. Nat. Rev. Genet.20, 207-220 https://doi.org/10.1038/s41576-018-0089-8 (2019).

43. Ziegenhain, C. et al. Comparative analysis of single-cell RNA sequencing methods. Mol. Cell.65, 6316434 https://doi.org/10.1016/j.molcel.2017.01.023 (2017).

44. Paik, D. T. et al. J. C. Wu. Large-scale single-cell RNA-seq reveals molecular signatures of heterogeneous populations of human induced pluripotent stem cell-derived endothelial cells. Circ. Res.123, 443-450 https://doi.org/10.1161/CIRCRESAHA.118.312913 (2018).

45. Chu, L. F. et al. J. A. Thomson. Single-cell RNA-seq reveals novel regulators of human embryonic stem cell differentiation to definitive endoderm. Genome. Biol.17, 173 https://doi.org/10.1186/s13059-016-1033-x (2016).

46. Hojo, H., Ohba, S., He, X., Lai, L. P. \& McMahon, A. P. Sp7/Osterix is restricted to bone-forming vertebrates where it acts as a Dlx co-factor in osteoblast specification. Dev. Cell.37, 238-253 https://doi.org/10.1016/j.devcel.2016.04.002 (2016).

47. Kim, D. et al. TopHat2: accurate alignment of transcriptomes in the presence of insertions, deletions and gene fusions. Genome. Biol.14, R36 https://doi.org/10.1186/gb-2013-14-4-r36 (2013).

48. Buenrostro, J. D., Giresi, P. G., Zaba, L. C., Chang, H. Y. \& Greenleaf, W. J. Transposition of native chromatin for fast and sensitive epigenomic profiling of open chromatin, DNA-binding proteins and nucleosome position. Nat. Methods.10, 1213-1218 https://doi.org/10.1038/nmeth.2688 (2013).

49. Langmead, B., Trapnell, C., Pop, M. \& Salzberg, S. L. Ultrafast and memory-efficient alignment of short DNA sequences to the human genome. Genome. Biol.10, R25 https://doi.org/10.1186/gb-200910-3-r25 (2009).

50. Jiang, H. J. H., Ma, W., Johnson, D. S. \& Myers, R. M. W. H. Wong. An integrated software system for analyzing ChIP-chip and ChIP-seq data. Nat. Biotechnol.26, 1293-1300 https://doi.org/10.1038/nbt.1505 (2008).

51. McLean, C. Y. et al. G. Bejerano. GREAT improves functional interpre- tation of cis-regulatory regions. Nat. Biotechnol.28, 495-501 https://doi.org/10.1038/nbt.1630 (2010).

\section{Figures}


a

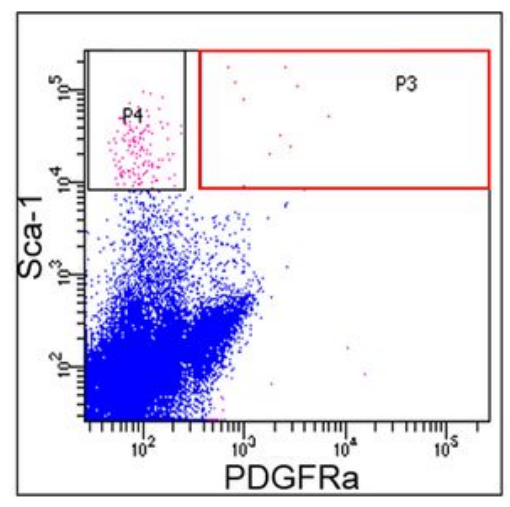

b

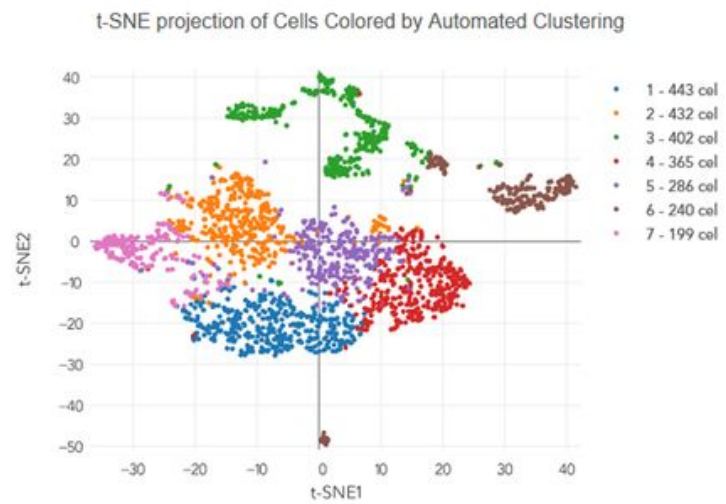

t-SNE Projection of Cells Colored by UMI Counts

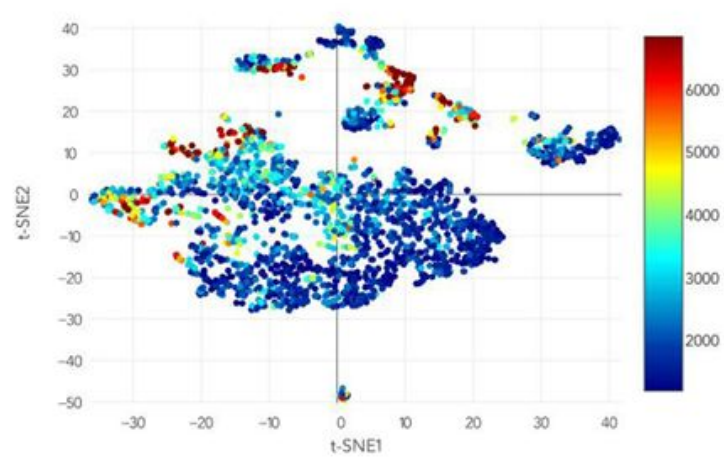

C

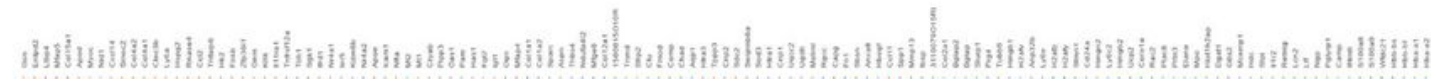

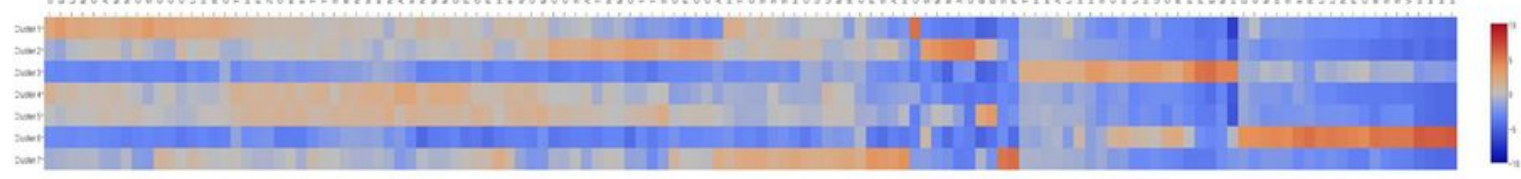

\section{Figure 1}

Single cell RNA-seq for the Pa-S fraction. (a) About 30,000 MSCs fractionated by PDGFRa, Sca-1 (Pa-S) were isolated from mouse bone marrow-derived mesenchymal cells. (b) MSCs isolated by Pa-S were analyzed with single-cell RNA-seq for high throughput expression profiling. t-SNE analysis detected genes of which expression varied among the clusters. (c) The gene expression pattern in each cluster was shown by a heat map. The color indicator represents UMI counts. 

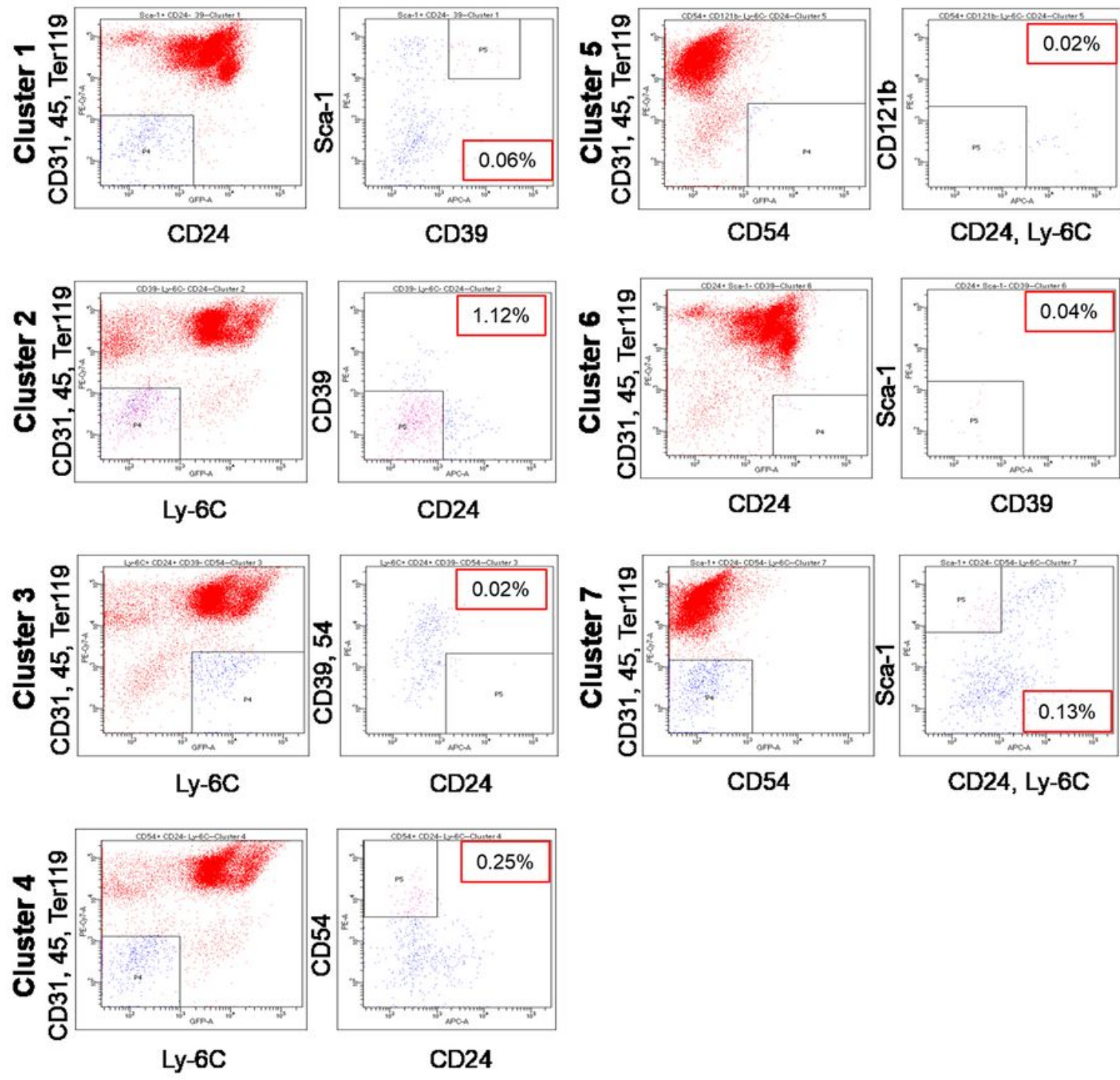

\section{Figure 2}

Isolation of each cluster using specific markers. Each cluster was isolated by using the surface antigen gene with large alteration in gene expression profiling by scRNA-seq of Pa-S. 
a

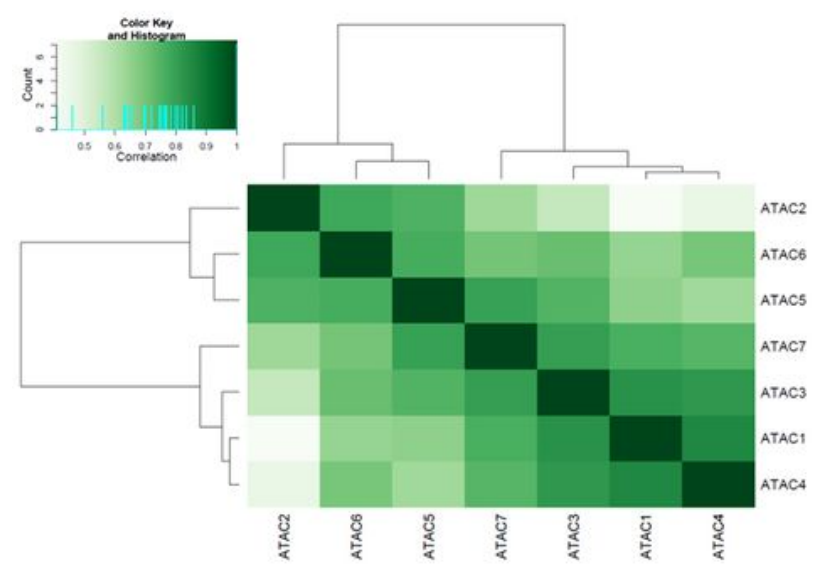

b

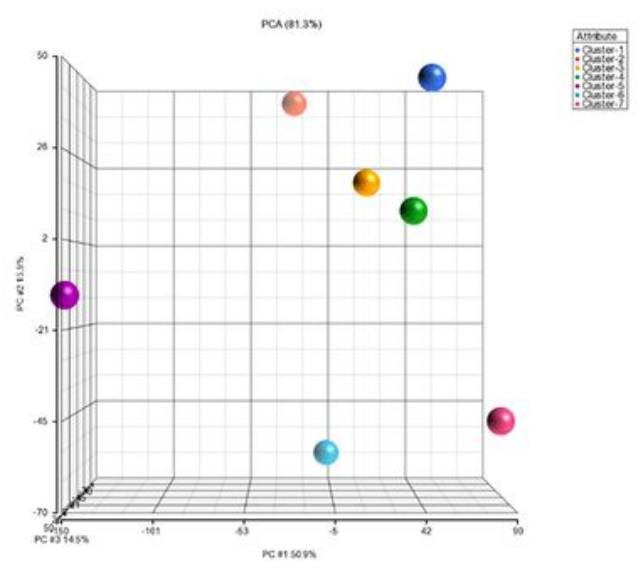

C

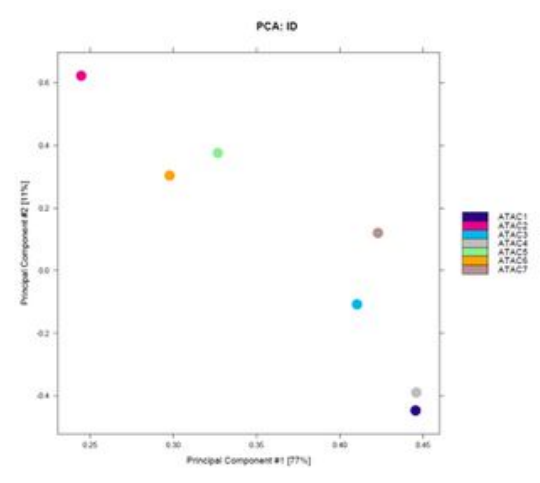

d Herarchical Custem
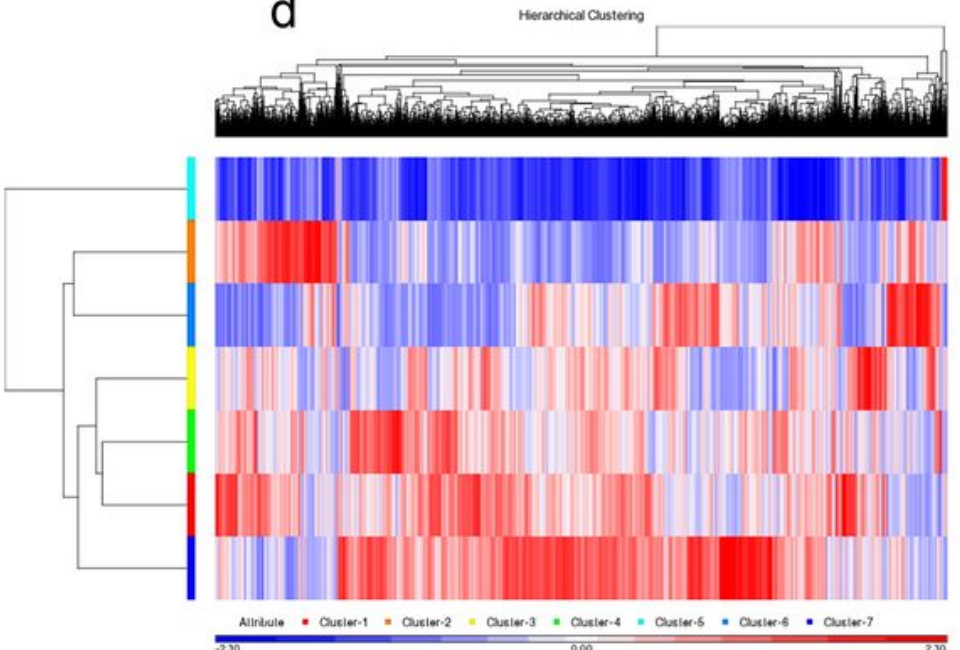

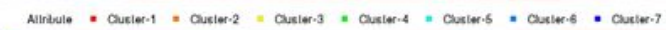

\section{Figure 3}

Identification of cluster-specific open chromatin regions and gene expression by ATAC-seq and RNA-seq analyses. (a) Clustering analysis for the peak intensity of each cluster in ATAC-seq. (b) PCA plots based on the peak intensity of ATAC-seq. c) PCA plot analysis based on the gene expression profiles in RNA-seq analysis. (d) Clustering analysis based on gene expression in RNA-seq analysis. 
a

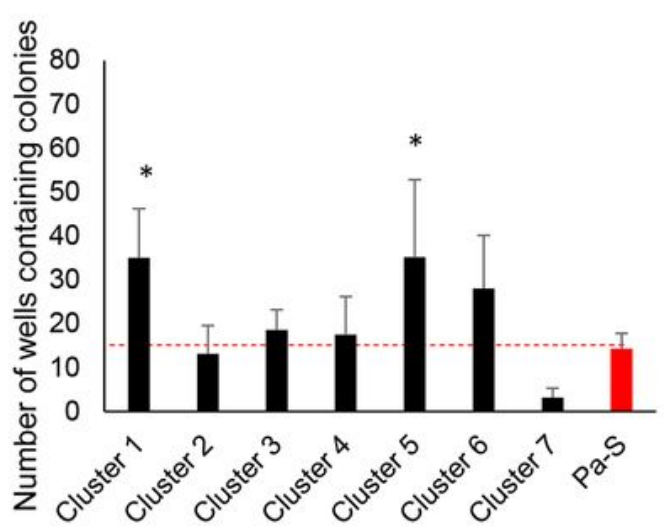

b

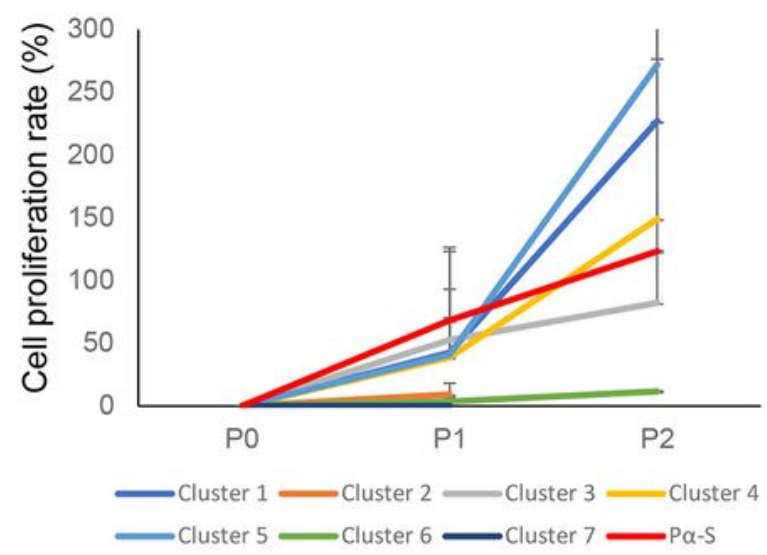

C
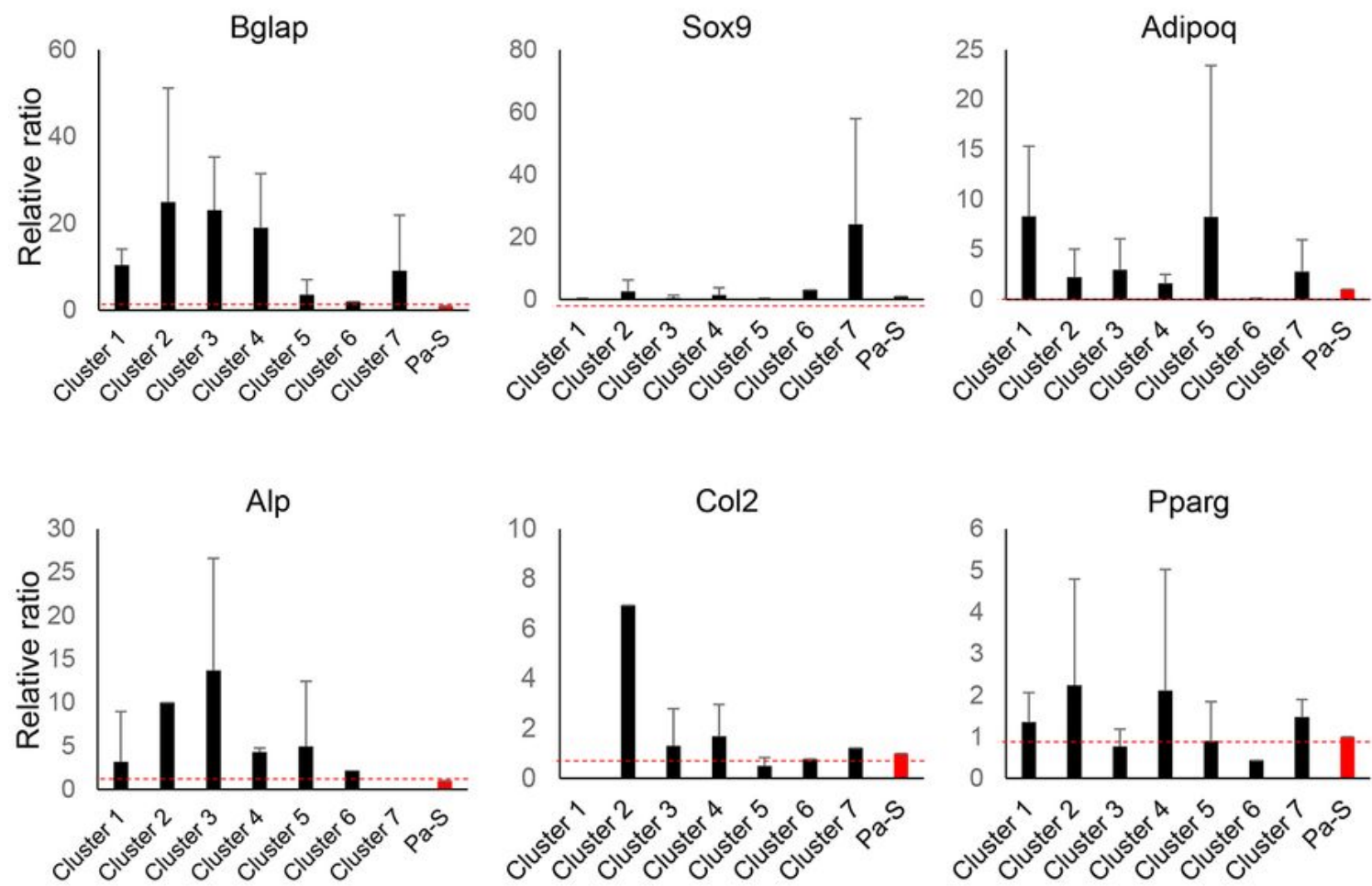

Pparg

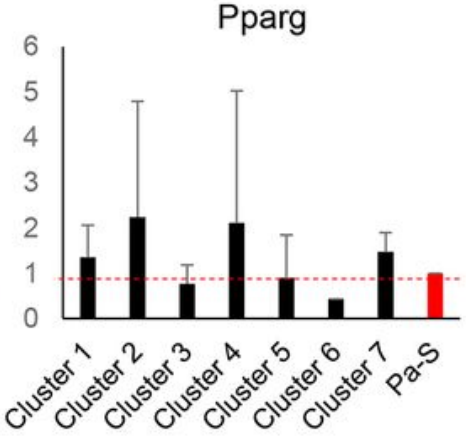

\section{Figure 4}

Stem cell properties of each cluster. (a) Graph showing bone marrow fibroblastic colony forming unit (CFU-F). ${ }^{*}<0.01$ vs Pa-S ( $n=9$ ). (b) Cell proliferation assay for each cluster. (c) Real Time PCR analysis for mesenchymal lineage marker expression in each cluster following 2-week treatment with mesenchymal differentiation media. ${ }^{\star *} \mathrm{P}<0.05$ vs $\mathrm{Pa}-\mathrm{S}(\mathrm{n}=6)$. 

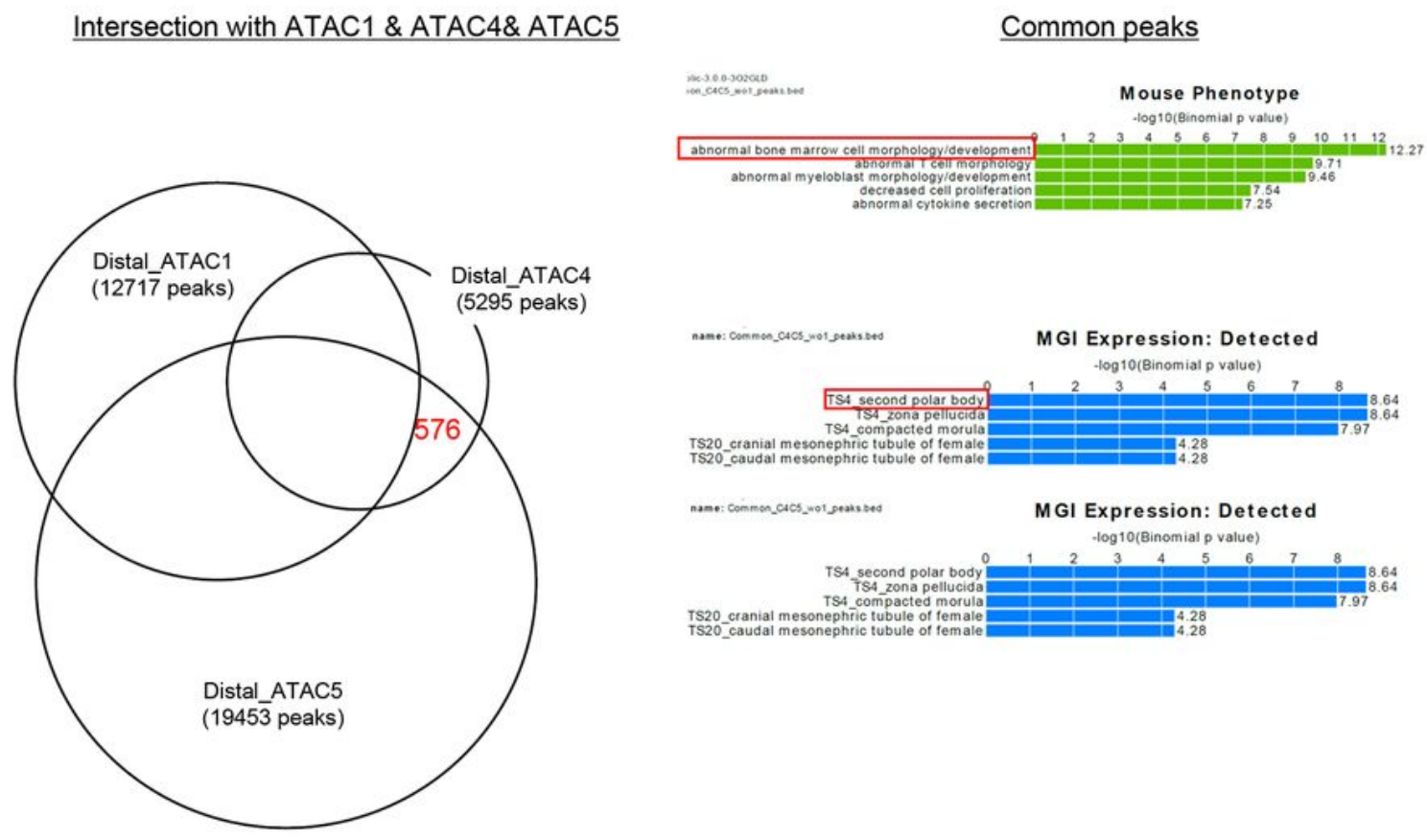

\section{Figure 5}

Analysis of open chromatin signals. In the ATAC-seq analysis, focusing on regions common to clusters 4 and 5 and different from cluster 1 , genes listed as genes associated with terms obtained by GREAT analysis and their expression were listed.

\section{Supplementary Files}


This is a list of supplementary files associated with this preprint. Click to download.

- 20201015supplementaltext.docx 